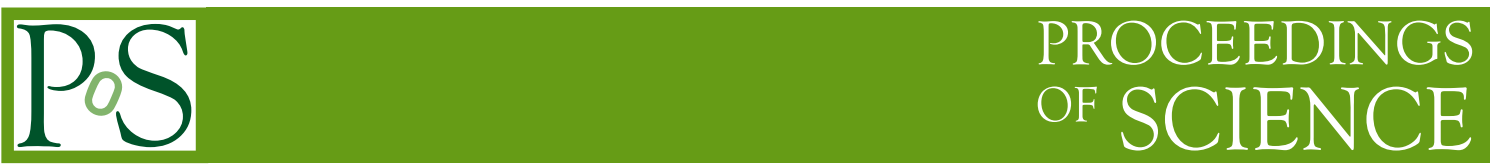

\title{
Electron identification with the ATLAS detector
}

\author{
Grigore TARNA, on behalf of the ATLAS Collaboration \\ IFIN-HH Bucharest (RO) / CPPM, Aix-Marseille Université, CNRS/IN2P3 (FR) \\ E-mail: grigore.tarnaecern.ch
}

\begin{abstract}
Electron identification is a crucial input to many ATLAS physics analysis. The electron identification used in ATLAS for run 2 is based on a likelihood discrimination to separate isolated electron candidates from candidates originating from photon conversions, hadron misidentification and heavy flavor decays. In addition, isolation variables are used as further handles to separate signal and background. The measurement of the efficiencies of the electron identification and isolation cuts are performed with the data using tag and probe techniques with large statistics sample of $Z \rightarrow e e$ and $J / \psi \rightarrow e e$ decays. These measurements performed with pp collisions data at $\sqrt{s}=13$ $\mathrm{TeV}$ in 2016 (2015) corresponding to an integrated luminosity of 33.9 (3.2) $\mathrm{fb}^{-1}$ of $\sqrt{s}=13 \mathrm{TeV}$ pp are presented.
\end{abstract}

The European Physical Society Conference on High Energy Physics

5-12 July, 2017

Venice 


\section{Introduction}

The ATLAS detector [1] is a general purpose detector at CERN's Large Hadron Collider (LHC). It consists of the following subdetectors: the Inner Detector (Pixel, Silicon Microstrip (SCT) and Transition Radiation Tracker (TRT)), the Lead-liquid argon electromagnetic (EM) calorimeter, the Hadronic and Forward calorimeters and the Muon system.

\section{Electron reconstruction and identification}

Electron ${ }^{1}$ reconstruction consists in fitting a track using information from the Inner Detector and matching it to an energy cluster in the EM calorimeter.

For the electron candidates passing reconstruction electron identification (ID) algorithms are applied in order to discriminate between prompt, isolated electron candidates (signal) from backgroundlike objects coming from photon conversions, hadron misidentification and heavy flavor decays. A schematic view of the electron reconstruction and identification and also the subdetectors involved is given in Figure 1.

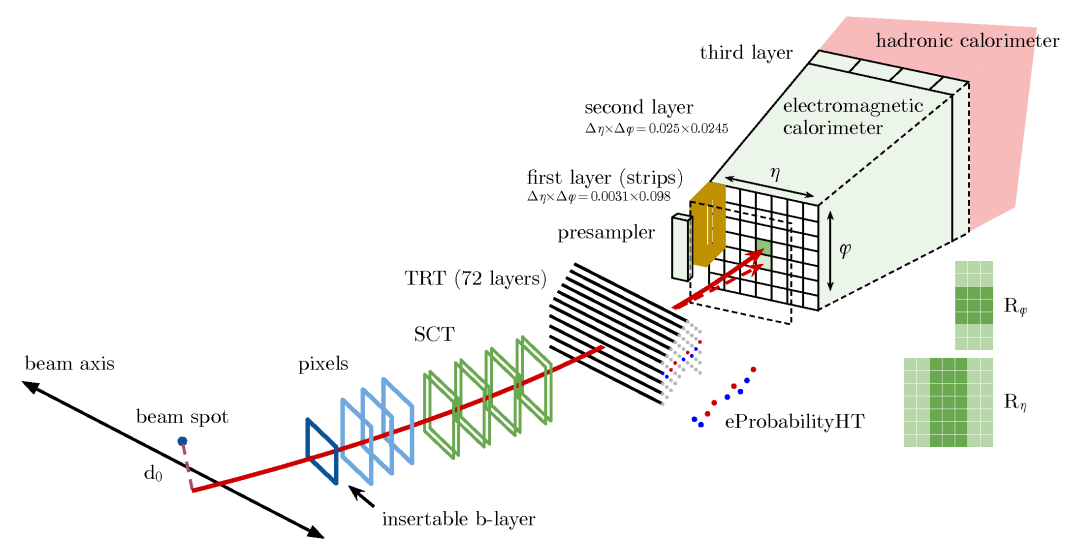

Figure 1: Schematic view of the electron reconstruction and identification [2]

The ID algorithm is based on a multivariate likelihood discriminant which is built using shower shape, track and track-to-cluster matching discriminating variables, with some Monte Carlo (MC) variables being shifted and scaled to reach the efficiency in data. Three levels of identification operating points are typically provided for electron ID. These are referred to, in order of increasing background rejection, as Loose, Medium, and Tight. The operating points are defined such that the set of electron candidates with higher background rejection is a subset of the electron candidates with lower background rejection (Tight $\subset$ Medium $\subset$ Loose).

\section{Efficiency measurement}

The measurement of the electron efficiencies is performed with a "Tag and Probe" method using large samples of electrons selected in $Z \rightarrow e e\left(E_{T}>15 \mathrm{GeV}\right)$ and $J / \psi \rightarrow e e\left(E_{T}<15 \mathrm{GeV}\right)$

\footnotetext{
${ }^{1}$ throughout this text the term "electron" refers to both electrons and positrons
} 
events. One electron in the event is required to pass strict selection conditions ("tag") while the other electron ("probe") is left unbiased.

Only the "probe" electrons are used in the measurement after a proper background subtraction.

- $Z \rightarrow e e$ : Two methods are used for background subtraction, illustrated in Figure 2. The first method uses the invariant mass of the tag-probe pair as a discriminating variable while the second method uses the isolation variable of the "probe" electron (signal like electrons are expected to be more isolated from other activities in the detector). Both methods are included in the measurement by considering them systematic variations.

- $J / \psi \rightarrow e e:$ The $J / \psi$ sample has a prompt and a non-prompt component and the displacement information $(\tau)$ between the $J / \psi$ vertex and the primary vertex is used to estimate the background with two methods. One method uses a cut on $\tau$ to enrich the prompt $J / \psi$ sample and subtract the background using the tag-probe invariant mass. In the second method the full $J / \psi$ sample is used and the background is estimated from fitting $\tau$.
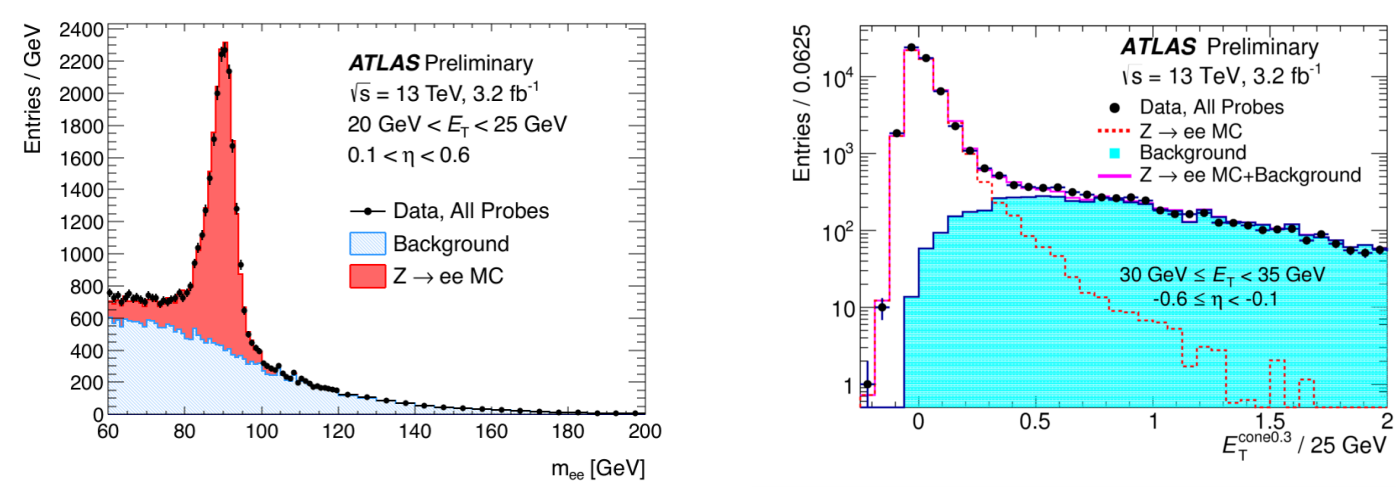

Figure 2: Background estimation using the invariant mass of the tag-probe pair (left) and background estimation using probe isolation as discriminating variable (right) [2].

Efficiency ratios are used in physics analysis to correct the MC efficiency to the efficiency measured in data.

\section{Results}

The combined electron reconstruction and ID efficiencies for 2015 (2016) data is given in Figure 3 (4), in the left plot as a function of $E_{T}$, inclusive in $\eta$, and in the middle plot as a function of $\eta$, inclusive in $E_{T}$. The lower efficiency in data than in $\mathrm{MC}$ arises from the fact that the MC does not properly represent the 2016 TRT conditions, in addition to the known mismodelling of calorimeter shower shapes in the GEANT4 detector simulation. The limits of the measurement using 2016 data are extended in transverse energy $(7.5 \rightarrow 4.5 \mathrm{GeV}$ and $80 \rightarrow 150 \mathrm{GeV})$ due to larger dataset available $(\approx \times 10)$. In Figure $3(4)$, right, the electron ID efficiency is given as a function of number of primary vertices. 

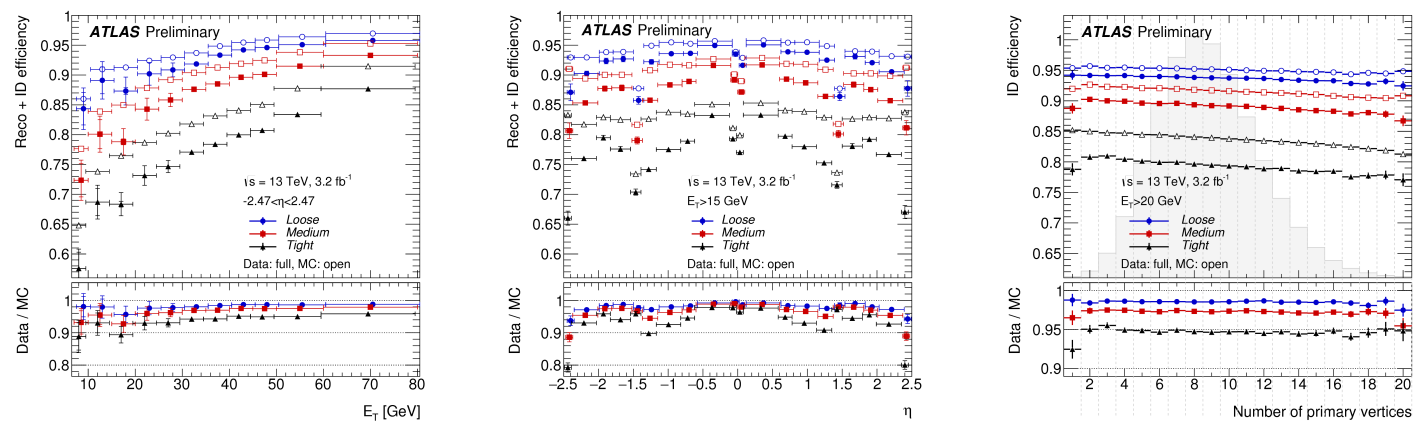

Figure 3: Electron reconstruction + ID efficiencies as a function of transverse energy $E_{T}$ (left) and pseudorapidity $\eta$ (middle). Electron ID efficiency as function of the number of reconstructed primary vertices (right). The results correspond to 2015 data $\left(3.2 \mathrm{fb}^{-1}\right)$ [2].
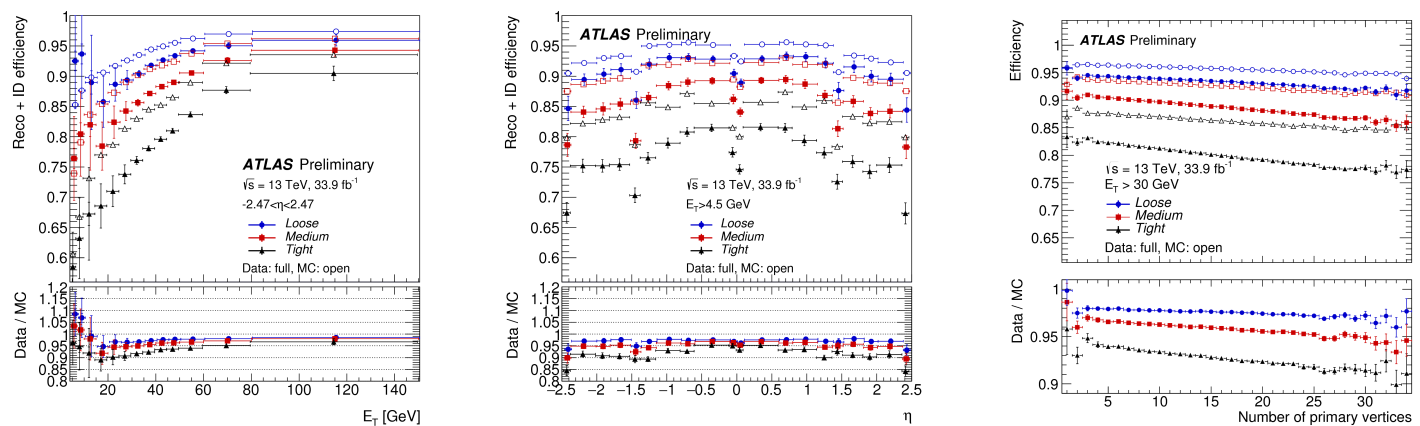

Figure 4: Electron reconstruction + ID efficiencies as a function of transverse energy $E_{T}$ (left) and pseudorapidity $\eta$ (middle) [3]. Electron ID efficiency as function of the number of reconstructed primary vertices (right) [4]. The results correspond to 2016 data $\left(33.9 \mathrm{fb}^{-1}\right)$.

\section{Conclusions}

The results for electron reconstruction and ID efficiencies measurement were presented for 33.9 (3.2) $\mathrm{fb}^{-1}$ data collected in 2016 (2015) by the ATLAS experiment. The precision of the measurement is around few percent at low $E_{T}$ and better than one per-cent for $E_{T}>30 \mathrm{GeV}$. High efficiency is retained despite harsh pileup environments.

\section{References}

[1] ATLAS Collaboration, The ATLAS Experiment at the CERN Large Hadron Collider, JINST 3 (2008) S08003.

[2] ATLAS Collaboration, Electron efficiency measurements with the ATLAS detector using the 2015 LHC proton-proton collision data, ATLAS-CONF-2016-024, 2016. https://cds.cern.ch/record/2157687

[3] ATLAS Collaboration, EGAM-2017-003, 2017. https://atlas.web.cern.ch/Atlas/GROUPS/PHYSICS/PLOTS/EGAM-2017-003/index.html

[4] ATLAS Collaboration, EGAM-2016-005, 2016. https://atlas.web.cern.ch/Atlas/GROUPS/PHYSICS/PLOTS/EGAM-2016-005/index.html 